Zirconium and Niobium Transmission Data at Wavelengths from 11-16 nm and 200-1200 nm

T.A. Johnson, R. Soufli, E.M. Gulikson, M. Clift

September 20, 2004

Optical Constants of Materials for UV to X-Ray Wavelengths (part of SPIE's Annual Meeting on Optical Science and Technology)

Denver, CO, United States

August 4, 2004 through August 5, 2004 
This document was prepared as an account of work sponsored by an agency of the United States Government. Neither the United States Government nor the University of California nor any of their employees, makes any warranty, express or implied, or assumes any legal liability or responsibility for the accuracy, completeness, or usefulness of any information, apparatus, product, or process disclosed, or represents that its use would not infringe privately owned rights. Reference herein to any specific commercial product, process, or service by trade name, trademark, manufacturer, or otherwise, does not necessarily constitute or imply its endorsement, recommendation, or favoring by the United States Government or the University of California. The views and opinions of authors expressed herein do not necessarily state or reflect those of the United States Government or the University of California, and shall not be used for advertising or product endorsement purposes.

This work was performed under the auspices of the U. S. Department of Energy by University of California, Lawrence Livermore National Laboratory under Contract w-7405-Eng-48 


\title{
Zirconium and niobium transmission data at wavelengths from 11-16 nm and 200-1200 nm
}

\author{
Terry A. Johnson*a, Regina Soufli ${ }^{\mathrm{b}}$, Eric M. Gullikson ${ }^{\mathrm{c},}$ Miles Clift $^{\mathrm{a}}$ \\ ${ }^{a}$ Sandia National Laboratories, 7011 East Avenue, Livermore, CA USA 94550 \\ ${ }^{\mathrm{b}}$ Lawrence Livermore National Laboratory, 7000 East Avenue, Livermore, CA USA 94550 \\ 'Lawrence Berkeley National Laboratory, 1 Cyclotron Road, Berkeley, CA USA 94720
}

\begin{abstract}
Transmission measurements of niobium and zirconium at both extreme-ultraviolet (EUV) and ultraviolet, visible, and near infrared (UV/Vis/NIR) wavelengths are presented. Thin foils of various thicknesses mounted on nickel mesh substrates were measured, and these data were used to calculate the optical constants $\delta$ and $\beta$ of the complex refractive index $n=1-\delta+i \beta$. $\beta$ values were calculated directly from the measured transmittance of the foils after normalizing for the nickel mesh. The average $\beta$ values for each set of foils are presented as a function of wavelength. The real (dispersive) part of the refractive index, $\delta$ was then calculated from Kramers-Kronig analysis by combining these $\beta$ values with those from previous experimental data and the atomic tables.
\end{abstract}

Keywords: Optical filters, optical constants, zirconium, niobium, extreme ultraviolet lithography, EUVL

\section{INTRODUCTION}

Extreme Ultra-Violet Lithography (EUVL), which has emerged as the next generation technology for IC manufacturing, may require the use of a spectral purity filter. The role of such a filter is to provide high throughput of energy from the EUV source at the desired wavelength while rejecting other energy ranges. UV, visible, and IR wavelengths can be reflected by EUV optics and cause unwanted mirror heating or exposure of the EUV resist.

As part of the EUVL program at Sandia National Laboratories (SNL), a spectral purity filter was developed for the Engineering Test Stand (ETS) ${ }^{1,2,3}$. The ETS is a full-field, alpha-class, step and scan EUVL tool that has been developed by SNL, Lawrence Livermore National Laboratory (LLNL), and Lawrence Berkeley National Laboratory (LBNL). The ETS has served as the basis for first-generation tool development by the commercial sector. The major components of the ETS are shown in Figure 1. Also shown is the location for the placement of a 4" diameter, mesh-supported, zirconium spectral filter manufactured by Luxel Corporation. The filter is located just downstream of the third set of mirrors in the condenser optical train. For the ETS, the spectral purity filter not only removed out-of-band radiation, but it also served as an environmental barrier between the two vacuum chambers that comprised the system. This was to limit the EUV absorption of the xenon gas used for the laser-produced plasma source.

In developing the spectral purity filter for the ETS, numerous combinations of thin metal foils and substrate materials were tested. Metals such as yttrium, beryllium, niobium, and zirconium were vacuum deposited on substrates of silicon and polyimide or epoxied to nickel mesh. A battery of tests was performed on these filters to

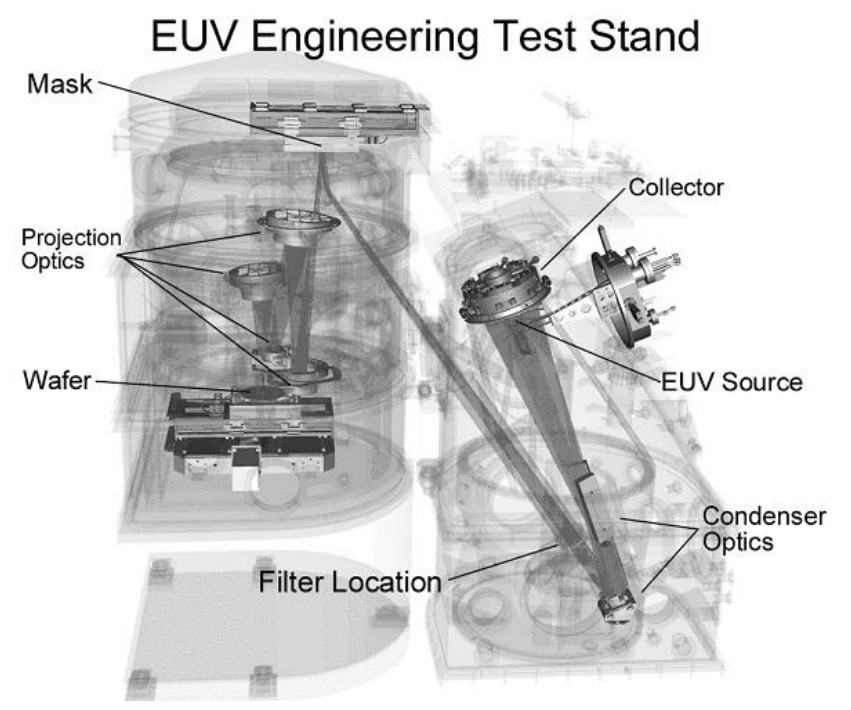

Figure 1. The EUV Engineering Test Stand

*tajohns@ sandia.gov; phone 1925 294-2512; fax 1925 294-3870 
determine their viability for use in the ETS. Tests included EUV transmittance, UV/Vis/NIR transmittance, Auger Electron Spectroscopy (AES), environmental aging, thermal, radiation, and burst pressure testing.

The results from the transmittance testing of niobium and zirconium filters are presented here. Additionally, calculations of the refractive index $(n=1-\delta+i \beta)$ of these materials were made based on the transmission data. Knowledge of the refractive index of niobium and zirconium could be important not only for EUVL applications, but for synchrotron research, solar astronomy, and x-ray microscopy, and spectroscopy. To our knowledge, these are the first published results of the transmission of zirconium in the 200-1200 nm energy region.

\section{EXPERIMENT}

\subsection{Sample Preparation}

The results presented here are all for filters fabricated by Luxel Corporation. Both niobium and zirconium foils were produced by vacuum electron beam deposition with ion assist to lower residual stress in the submicron foils. Also, care was taken to minimize the oxidation of the foils both during deposition and filter processing. The effect of oxidation is to decrease EUV transmittance and increase visible light transmittance, both of which are detrimental for lithography applications.

To produce a freestanding filter, first the metal foils were deposited on a coated substrate. A wet chemical process was then used to remove the foils from the substrate. Metal foils this thin need support to withstand handling. Thus, following deposition, the $19 \mathrm{~mm}$ diameter foils were epoxied to a commercially available nickel mesh for structural support. The 70 line-per-inch nickel mesh is produced by Buckbee Mears using an electroforming process and has an effective transmittance of up to $82 \%$.

As a final step, the filters were then attached to a metal frame to assist in handling and mounting of the filters. Figure 2 shows a photo of one of the zirconium filters produced by Luxel. All of the filters received were accompanied by a certification sheet. These sheets listed the metal thickness, manufacturer, type, and lot number; the mesh type, manufacturer, and transmission; the adhesive manufacturers and lot numbers; and the frame type. All thicknesses were measured using a surface profilometer and quoted as $\pm 50 \AA$.

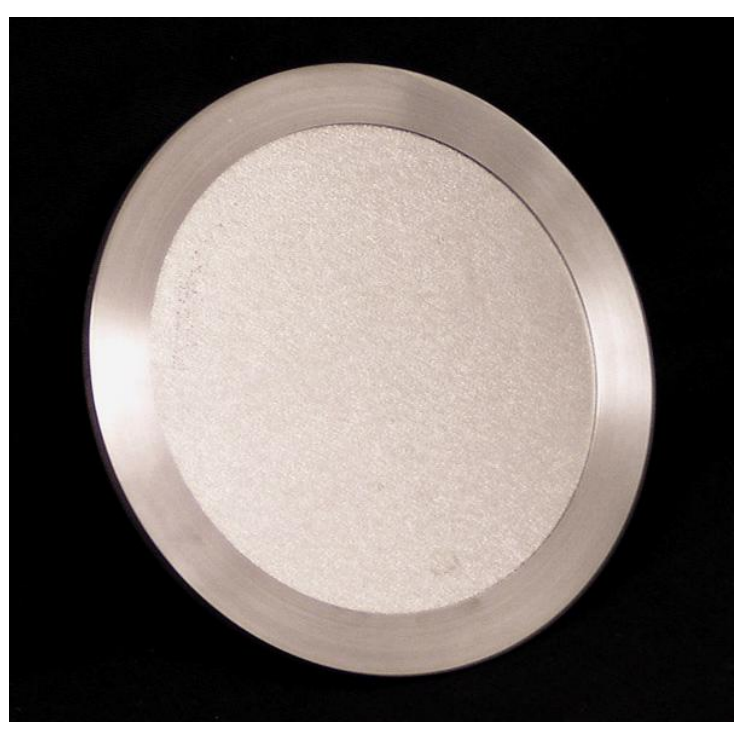

Figure 2. Photo of a zirconium/mesh spectral purity filter

\subsection{Measurement Apparatus}

The instrument used to measure the EUV transmittance of the filters is the EUV/soft $\mathrm{x}$-ray reflectometer at Lawrence Berkeley National Laboratory (LBNL) ${ }^{4}$. In brief, the reflectometer uses a laser-produced plasma from a gold target and a spherical grating monochromator. Continuously varying wavelengths from $4-41$ $\mathrm{nm}$ can be scanned by the monochromator. The beam passes from the monochromator through an adjustable exit slit and is sampled by an $\mathrm{I}_{0}$ detector used to normalize for shot-to-shot noise. A number of optical filters can be chosen to block the second harmonic of the beam prior to entering the reflectometer chamber. Samples are mounted in the chamber on a three degree-of-freedom positioning stage. The filters were positioned to intercept the beam at normal incidence. A sensor directly behind the filter then measured the transmitted energy. The entire system is kept at vacuum levels by a cryogenic pump.

The attenuation of the filters at non-EUV wavelengths was measured with a Perkin-Elmer UV/Vis/NIR spectrometer at SNL. The Lambda 9 model is a double monochromator spectrometer, which uses tungsten-halogen and deuterium sources to encompass a measurement wavelength range of 185-3200 nm. The region from 185 to $200 \mathrm{~nm}$ requires a nitrogen purge. The instrument incorporates a "pencil beam" with a uniform 
$10 \mathrm{~mm}$ X $2 \mathrm{~mm}$ size. The spectral bandwidth is adjustable down to $0.05 \mathrm{~nm}$ in the UV/Vis region and $0.2 \mathrm{~nm}$ in the NIR. The wavelength accuracy of the system is $0.2 \mathrm{~nm}$ in the UV/Vis region and $0.8 \mathrm{~nm}$ in the NIR.

The purity of both the zirconium and niobium filters was established using Auger Electron Spectroscopy (AES) at SNL. The Auger system used was a Physical Electronics Model 660 with typical e-beam parameters of $5 \mathrm{keV} 80 \mathrm{nA}$ rastered over a $30 \mu \mathrm{m}$ × $30 \mu \mathrm{m}$ area. Sputter Auger profiling was done using a $2 \mathrm{keV} 30 \mathrm{nA} \mathrm{Ar}^{+}$ion beam at a $45^{\circ}$ angle of incidence rastered over a $300 \mu \mathrm{m} \times 300 \mu \mathrm{m}$ area. A linear-least-squares fit was applied to all Auger data to reduce noise and to differentiate between types of carbon.

All depth scales for the Auger depth profiles, which should be used for comparison only, were adjusted to fit a standard $1000 \AA \mathrm{Si}$ oxide sputter rate. No attempt was made to more explicitly correct the depth axis for the elements being sputtered. Carbon plotted separately as carbide could be carbon intermixed with the metal since carbides can form during ion bombardment when some carbon species are present.

\section{RESULTS}

\subsection{Auger analysis/filter purity}

The zirconium transmission data reported in this manuscript came from measurements of three $100 \mathrm{~nm}$ thick filters that were the result of development efforts over several years at SNL and Luxel. Due to the destructive nature of the procedure, these filters were not analyzed by Auger. However, Figure 3 shows AES data for a zirconium filter of $100 \mathrm{~nm}$ thickness that was fabricated earlier in the development process. The plot shows the average of two Auger depth profiles taken at different locations on the filter. As will be discussed in section 3.2, the oxygen and carbon contamination shown in Figure 3 is likely higher than that in the filters reported on here.

As mentioned previously, the depths shown in the plot are not exact, yet the overall thickness of the foil is approximately correct. The oxide on the surface of the metal is most likely less than $100 \AA$ thick. There seems to be

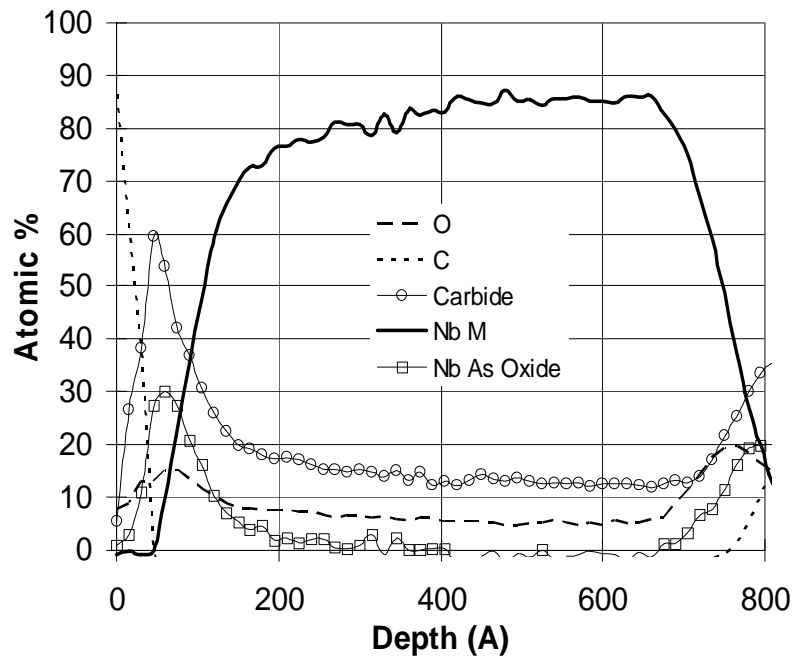

Figure 4. Auger depth profile of $66.2 \mathrm{~nm}$ thick niobium filter.

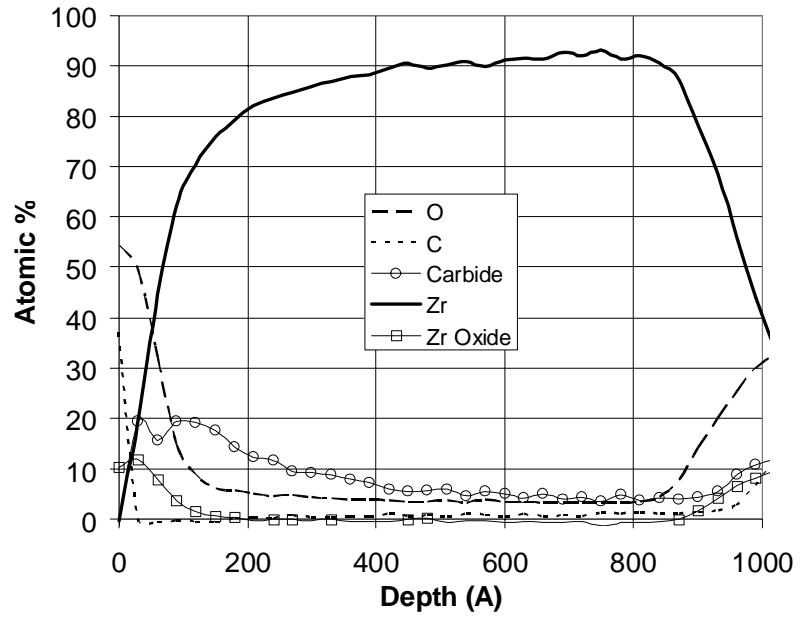

Figure 3. Auger depth profile of $100 \mathrm{~nm}$ thick zirconium filter.

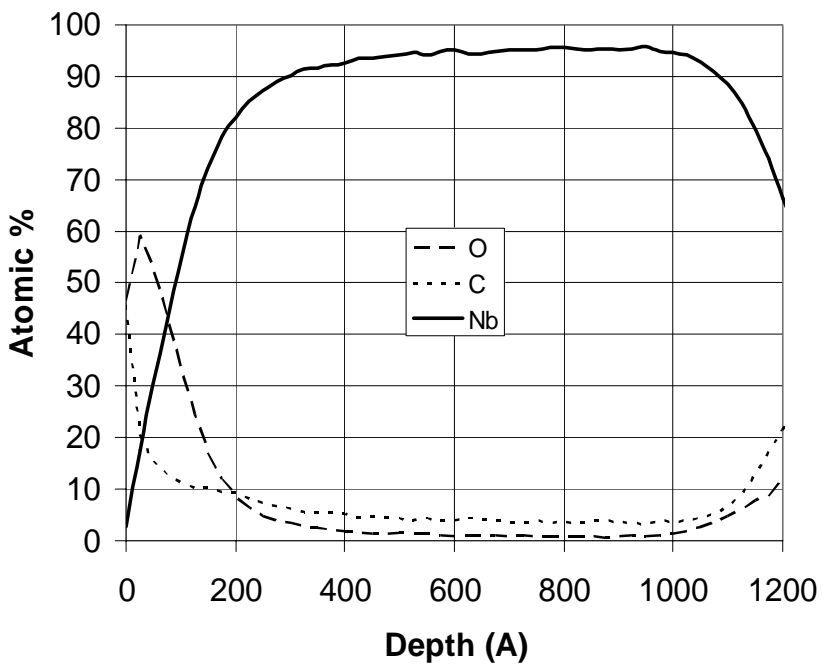

Figure 5. Auger depth profile of $114 \mathrm{~nm}$ thick niobium filter. 
some carbon present throughout the foil whether in the form of carbide or carbon mixed with the zirconium. These contaminants reduce the EUV transmittance of the filter. It can be inferred from a transmittance comparison (see Figure 6) that the filters reported on here have less contamination and should provide more accurate optical constants.

Fortunately, the niobium filters used for this manuscript do have corresponding AES results. Two $66.2 \mathrm{~nm}$ thick $\mathrm{Nb}$ filters were measured for transmittance. Following those measurements, one of the filters was analyzed with Auger and those results are shown in Figure 4. Additionally, a filter of $114 \mathrm{~nm}$ thickness was analyzed following transmittance measurements. Figure 5 shows the results from the thicker filter.

As with the zirconium results, the estimated thicknesses from the Auger results seem to match the stated values. The $66.2 \mathrm{~nm}$ filters have more contamination based on these plots. Like the zirconium filter, these filters have carbon throughout the thickness. Both plots show about 100 angstroms of surface contamination. Though perhaps not as 'clean' as the zirconium filters, the niobium filters should also yield good results.

\subsection{Transmission measurements}

\subsubsection{EUV transmission}

Transmission measurements were made over EUV wavelengths from 10.8 to $15.8 \mathrm{~nm}$ for all of the zirconium and niobium filters. All filters were fabricated using 70 line-per-inch nickel mesh. Figures 6 and 7 show the transmittance results for these filters with the mesh normalized out.

Because they were the same thickness, the average transmittance of the three zirconium filters is shown in Figure 6(a). Figure 6(b) is the transmittance of the more contaminated filter whose Auger results are shown in Figure 3. The third plot in Figure 6 is the theoretical combination of (a) with $50 \AA$ of zirconium oxide. The zirconium oxide transmittance was found from the Center for X-ray Optics (CXRO) website. It can be seen that the combined transmittance shown in Figure 6(c) matches closely the transmittance of (b). Combined with Figure 3, this data gives a qualitative indication of the contamination levels in the three zirconium filters.

Figure 7 shows the niobium EUV transmission data. The results for the $114 \mathrm{~nm}$ thick filter are shown in (a), while the average of the two $66.2 \mathrm{~nm}$ thick filters is shown in (b). Note that the transmittance of the $66.2 \mathrm{~nm}$ thick filters is very similar to that of the $100 \mathrm{~nm}$ thick zirconium filters in Figure 6(a). The better EUV transmittance of zirconium was the reason it was chosen for the ETS spectral purity filter.

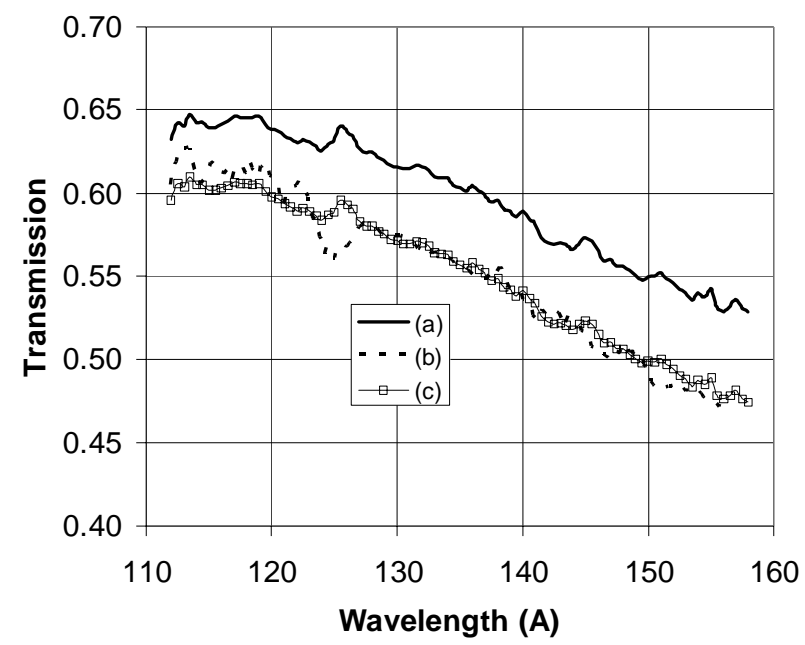

Figure 6. EUV transmission results for zirconium filters. (a) is the average of 'clean $100 \mathrm{~nm}$ filters, (b) is the more contaminated filter from Figure 3, and (c) is the combination of (a) with $50 \AA$ oxide.

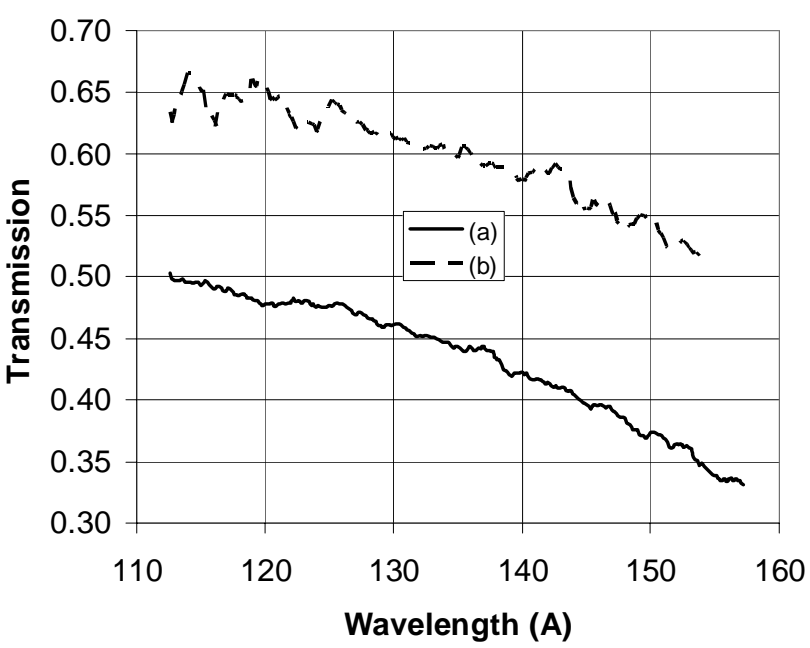

Figure 7. EUV transmission results for niobium filters. (a) is the average of two $66.2 \mathrm{~nm}$ thick filters and (b) is a $114 \mathrm{~nm}$ thick filter. 


\subsubsection{UV/Vis/NIR transmission}

Transmission measurements were made in the wavelength range of $200 \mathrm{~nm}$ to $1200 \mathrm{~nm}$ using the Perkin-Elmer instrument described in section 2.2. The low end of this range was set by the limitations of the spectrometer while the upper end was set to encompass the wavelength of the ETS laser at $1064 \mathrm{~nm}$. Figure 8 shows the transmission results for both the zirconium and niobium filters. The average of the three zirconium filters is shown in (a), the average for the $66.2 \mathrm{~nm}$ thick niobium filters is shown in (b), and the $114 \mathrm{~nm}$ thick niobium filter is shown in (c). The increased noise level above $850 \mathrm{~nm}$ is due to a change in the light source of the instrument.

A comparison of the niobium filters with the zirconium filters shows again why zirconium was chosen for the ETS. For filters of comparable EUV transmittance, the $66.2 \mathrm{~nm}$ thick niobium filters block much less out-of-band energy than the $100 \mathrm{~nm}$ thick zirconium filters.

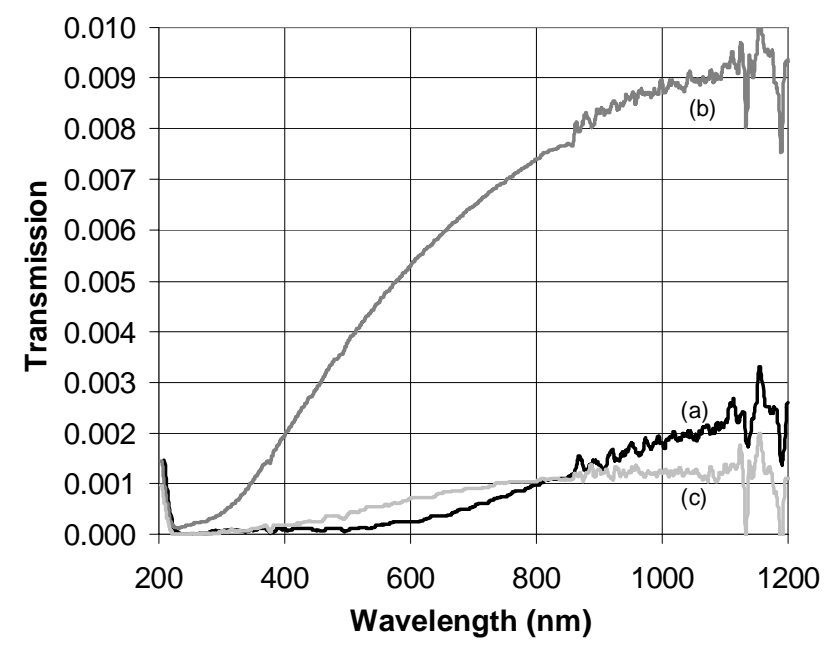

Figure 8. UV/Vis/NIR transmission results for (a) $100 \mathrm{~nm}$ thick zirconium, (b) $66.2 \mathrm{~nm}$ thick niobium, and (c) $114 \mathrm{~nm}$ thick niobium.

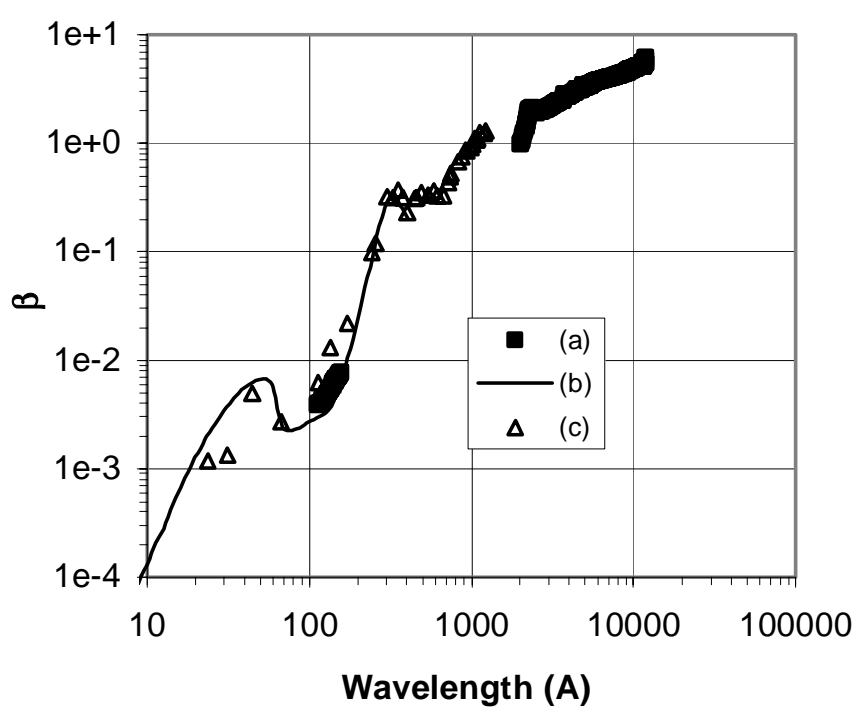

Figure 9. Zr beta values for (a) the measured EUV and UV/Vis/NIR transmittance, (b) CXRO tables, and (c) Windt et al. $^{7}$

\subsection{Absorption and dispersion results}

The determination from transmittance measurements of the optical constants $\delta$ and $\beta$ of the complex refractive index (n $=1-\delta+\mathrm{i} \beta$ ) has been described in detail by previous authors $^{5,6}$. Briefly, the absorptive part $\beta$ can be determined directly from the transmittance $\mathrm{T}$ with the equation

$$
\beta=\frac{-\lambda}{4 \pi x} \ln (T)
$$

where $\lambda$ is the wavelength of energy, and $x$ is the thickness of the material. The dispersive part of the refractive index can be calculated from the Kramers-Kronig relation

$$
\delta(E)=\frac{2}{\pi} \int_{0}^{\infty} \frac{E^{\prime} \beta\left(E^{\prime}\right)}{E^{2}-E^{\prime 2}} d E^{\prime},
$$

where $E=h c / \lambda$ is the photon energy. As equation (2) indicates, $\delta$ must be determined from an integral of $\beta$ over a semi-infinite energy range. Thus the $\beta$ values determined here were combined with values from the CXRO tables to produce dispersion values. However, because the cumulative errors at long wavelengths would produce unreliable results, $\delta$ was only calculated for EUV wavelengths. The fitted $\delta$ values were found to be nearly identical to those produced with the CXRO tables. Thus, only the $\beta$ values are plotted here.

Figures 9 and 10 show the results from applying equation (1) to the transmission values for the zirconium and niobium filters respectively. The calculated $\beta$ values for zirconium are shown in 9(a) along with values obtained from the CXRO tables (b) and previous experimental values (c) ${ }^{7}$ for zirconium. The new results in the EUV region fit well with the existing data. The new results in the $200-1200 \mathrm{~nm}$ wavelength range are the first to the authors' knowledge for this energy region. 


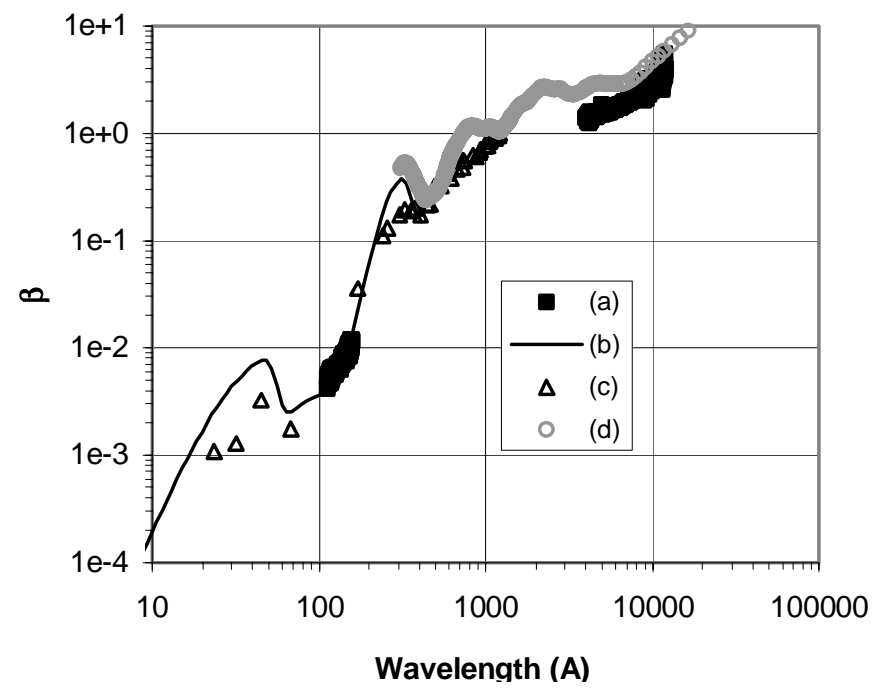

Figure 10. $\mathrm{Nb}$ beta values for (a) the measured EUV and UV/Vis/NIR transmittance, (b) CXRO tables, (c) Windt et al. ${ }^{7}$, and (d) Weaver et al. ${ }^{8}$
The new $\beta$ values for the niobium filters are shown in 10(a). Also shown are $\beta$ values for niobium from the CXRO tables (b), Windt et al. ${ }^{7}$ (c), and Weaver et al. ${ }^{8}$ (d). As with the zirconium results, the $\beta$ values in the EUV region complement the existing results. The longer wavelength results seem reasonable though somewhat lower than the $\beta$ values from Weaver's 1973 data. This may be due to the fact that Weaver's results were for single crystal niobium as compared to the thin films used here. Also, the results shown in (d) were from calorimetry, absorptivity and reflectivity measurements as compared to our transmittance measurements.

\section{CONCLUSIONS}

Optical filters were developed for the EUVL program at Sandia National Laboratories to provide a spectrally pure energy source to the projection optics of the ETS. Transmission measurements were made in both the EUV and UV/Vis/NIR wavelength regions, and were presented here for prototype filters of niobium and zirconium. Calculations were made from the transmittance data to determine the absorptive part $\beta$ of the complex refractive index. Values obtained for both materials fit well with existing data and calculated values from the CXRO tables. The zirconium data at the longer wavelengths represents the first published results in this region to our knowledge.

This work was supported by the Extreme Ultraviolet Limited Liability Corporation under a Cooperative Research and Development Agreement and by the U.S. Department of Energy under contract DE-AC04-94AL85000.

\section{REFERENCES}

1. F. R. Powell and T. A. Johnson, "Filter windows for EUV lithography," Proceedings of SPIE, 4343, 585-589, 2001.

2. D. A. Tichenor et al., "EUV Engineering Test Stand," Proceedings of SPIE, 3997, 48-69, 2000.

3. D. A. Tichenor et al., "System Integration and Performance of the EUV Engineering Test Stand," Proceedings of SPIE, 4343, 19-37, 2001.

4. E. M. Gullikson, J. H. Underwood, P. J. Batson, and V. Nikitin, "Soft X-ray/EUV reflectometer based on a laser produced plasma source," Journal of X-Ray Science and Technology, 3, 283-299, 1992.

5. B. Sae-Lao and R. Soufli, "Measurements of the refractive index of yttrium in the 50-1300-eV energy region," Applied Optics, 41, 7309-7316, 2002.

6. R. Soufli and E. M. Gullikson, "Absolute photoabsorption measurements of molybdenum in the range $60-930 \mathrm{eV}$ for optical constant determination," Applied Optics, 37, 1713-1719, 1998.

7. D. L. Windt et al., "Optical constants for thin films of Ti, Zr, Nb, Mo, Ru, Rh, Pd, Ag, Hf, Ta, W, Re, Ir, Os, Pt, and Au from $24 \AA$ to $1216 \AA$ A,” Applied Optics, 27, 246-278, 1988.

8. J. H. Weaver, D. W. Lynch, and C. G. Olson, “Optical Properties of Niobium from 0.1 to $36.4 \mathrm{eV}$," Physical Review B, 7, 4311-4318, 1973. 